\title{
Forming Nanoparticle Monolayers at Liquid-Air Interfaces by Using Miscible Liquids
}

\author{
Datong Zhang, Jiayang Hu, Kathleen M. Kennedy, and Irving P. Herman* \\ Department of Applied Physics and Applied Mathematics, \\ Columbia University, New York, NY 10027, USA
}

\section{Supporting Information}

\section{Intensity vs q Dependence for the in situ SAXS Pattern}

The in situ SAXS pattern of a monolayer of $13.2 \mathrm{~nm}$ iron oxide NPs on a tilted surface is shown in Fig. 2(b). The aspect ratio $q_{\mathrm{x}} / q_{\mathrm{y}}=\sin \alpha$ with $\alpha$ the tilt angle. The scattering wave vector is defined as $q_{h, k}=\sqrt{q_{x}^{2}+q_{y}^{2} \sin ^{2} \alpha}$, following Ref [1]. The intensity- $q_{\mathrm{h}, \mathrm{k}}$ relation is obtained by averaging the intensities of the points on the SAXS pattern with same $q_{\mathrm{h}, \mathrm{k}}$ values, and shown in Fig. S1. 


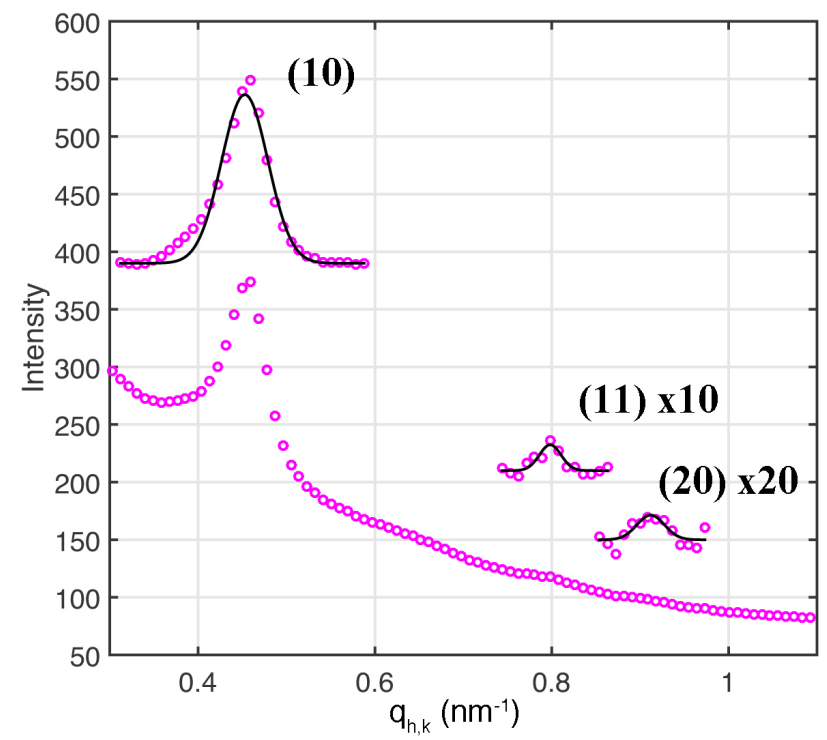

Figure S1. Averaged scattering intensities with respect to scattering wave vectors. Each peak is fitted by a Gaussian function after subtracting the background. The (11) and (20) peaks are multiplied by a factor of 10 and 20 .

\section{Ex situ SAXS Peak Fit Using the KTHNY Model}

Ex situ SAXS patterns (Figs. 3a and 3b) are fit with the KTHNY model. ${ }^{2,3}$ The structure factor is first obtained by dividing the ex situ SAXS intensity map using a calculated form factor map, for a particle size of $13.2 \mathrm{~nm}$ and a standard size distribution of $\pm 7.5 \%$. Then the (10) peak intensity is fit with a Kummer function numerically (Fig. $\mathrm{S} 2$ ), which gives an effective NP monolayer domain size of $\sim 200 \mathrm{~nm}$ and $\sim 100 \mathrm{~nm}$ for the immiscible and miscible solvent pair cases, respectively, with uncertainties of $\sim \pm 10$ $\mathrm{nm}$ each. (In the fitting procedure, the lateral position of the peak ( $g$ in the Kummer function) was optimized, the intensity was optimized to produce the same integrated area as the data, and the domain size was varied to reproduce the width for optimal values of the KTHNY decay exponent parameter $\eta$. For these the miscible and immiscible cases $\eta$ 
is 0.25 and 0.35 .) The domain size for the immiscible solvent pair case is comparable with that given in the literature, ${ }^{2}$ which is much smaller than those obtained when the film is not fully dried; fracture during drying decreases the measured domain size. For the miscible solvent pair case, the effective domain size is comparable with the domain sizes obtained after transfer and TEM analysis. This is probably due to the small single domain size of the film, so any new cracks that are formed in drying and transfer are generated along domain boundaries instead of within the domains.

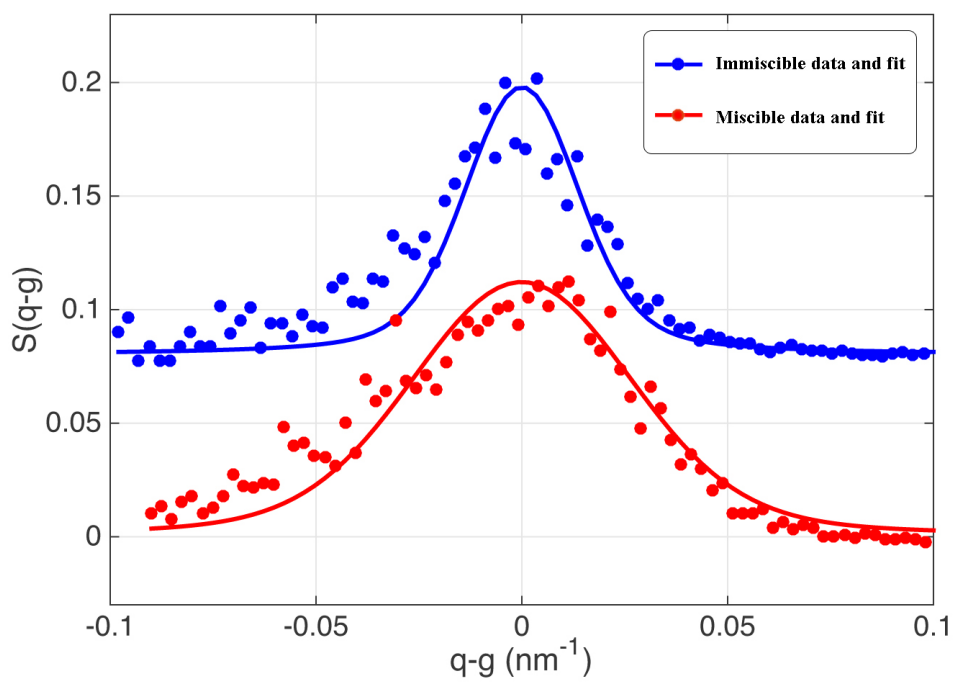

Figure S2. Curve fits for the (10) peak shape using the KTHNY model (the blue, immiscible solvent pair curve is offset vertically), which give effective domain sizes of $\sim 200 \mathrm{~nm}$ and $\sim 100 \mathrm{~nm}$ for the immiscible and miscible solvent pair cases, respectively.

\section{Test of NP Monolayer Uniformity}

Optical microscopy shows that a uniform monolayer forms on the DMSO surface when produced using miscible solvent pairs. The uniformity of the NP monolayer quality was investigated by scooping up TEM samples at five different places in a single run (Fig. S3), including near the center and edges of the same Petri dish, $24 \mathrm{~h}$ after 4 ML- 
equivalents of the toluene dispersion were drop-cast on the DMSO. Figure S4 shows the TEM images from these positions with relatively low and high magnifications (size bars are shown). We conclude from these and similar trials that the NP monolayer formed using miscible solvent pairs is generally uniform over the surface of the Petri dish.

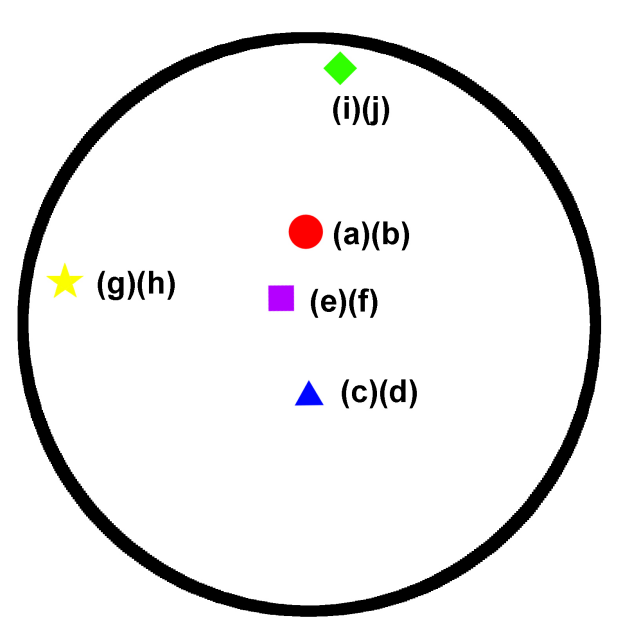

Figure S3. Locations in the Petri dish where samples were scooped up for TEM analysis, in Fig. S4. 


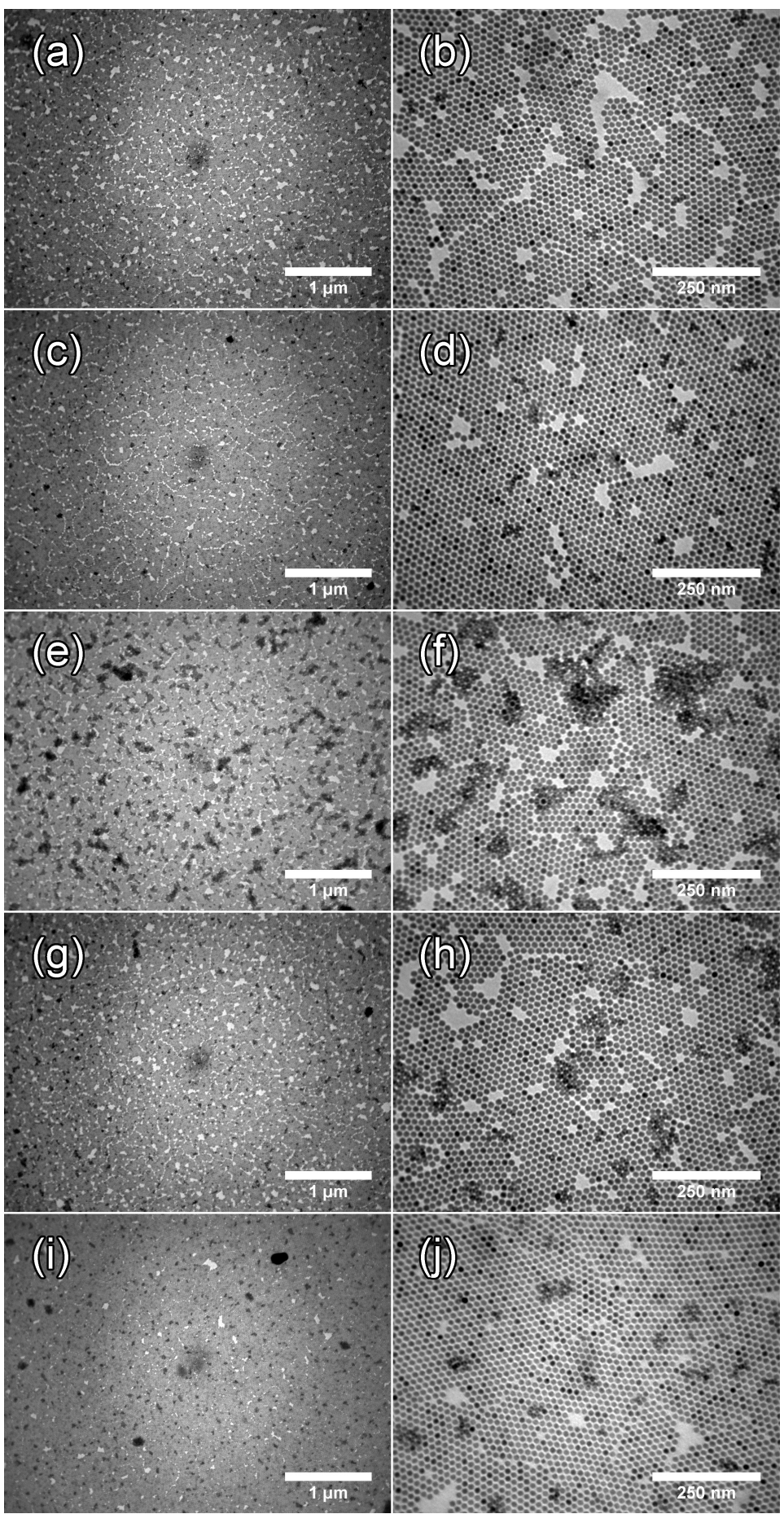

Figure S4. TEM images of NP monolayer sample prepared using the miscible solvent pair method, scooped at five different places $24 \mathrm{~h}$ after drop-casting 4 ML-equivalents of the toluene dispersion on the DMSO surface, at the locations shown in Fig. S3, with (a),

(c), (e), (g), and (i) at lower magnifications and (b), (d), (f), (h), and (j) for the same samples respectively, presented at higher magnification. 


\section{Energy Diagram of OA-capped NP at the Liquid-Air Interface}

The Pieranski model is used to analyze the binding of OA-capped $13.2 \mathrm{~nm}$ NPs to the liquid-air interface. ${ }^{4}$ Figure S5 plots the overall energy versus the vertical position of NP relative to the liquid surface for DEG, DMSO, DMF, ethanol, and acetone, using the surface tension energies of these liquids that are given in the main text. For $z / R=-1$ the $\mathrm{NP}$ is in the bulk liquid and its top just touches the liquid surface, while for $z / R=1$ the NP is in air and its bottom just touches the surface.

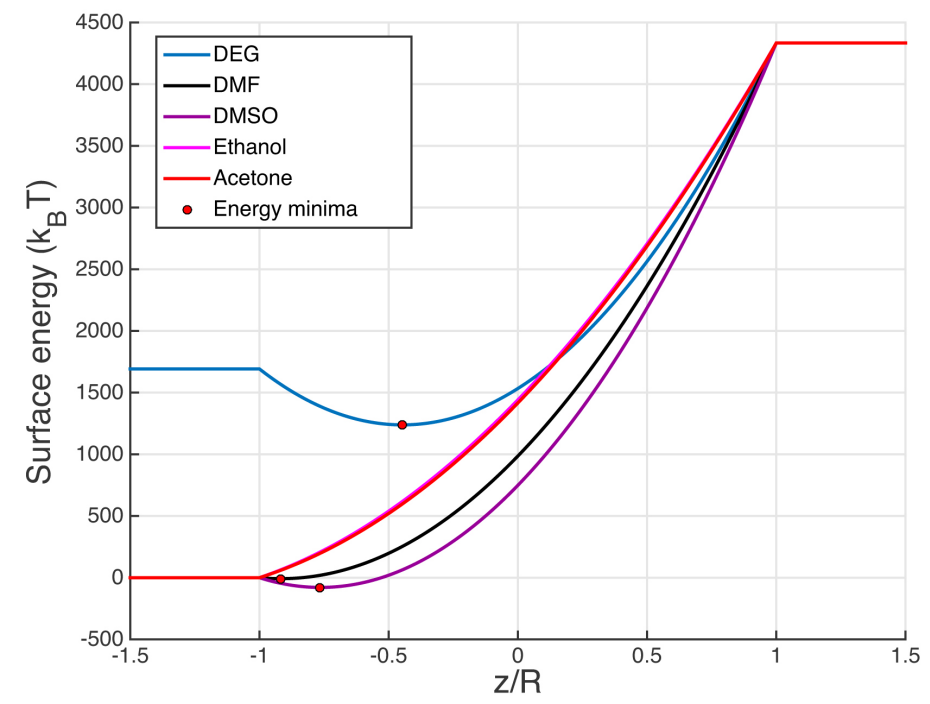

Figure S5. Energy of an OA-capped $13.2 \mathrm{~nm} \mathrm{NP}$ at several liquid-air interfaces, where $z$ is the distance of the center of the NP core to the liquid-air interface and $R$ is the NP radius. When there is an energy minimum it is denoted with a red dot.

\section{Extension to Other Miscible Pairs and Nanoparticles}

The generality of the miscible solvent pair method was tested with other miscible solvent pairs and NP systems. This method is seen to be transferrable to the benzene/DMSO system and fluorobenzene/DMSO system for the same batch of NPs as 
used in the main text. Figure $\mathrm{S} 6$ shows the TEM images of the $13.2 \mathrm{~nm}$ NP monolayer formed on the liquid surface when using these two systems. The monolayer quality and coverage are qualitatively similar to the monolayer prepared using the toluene/DMSO miscible solvent pair.
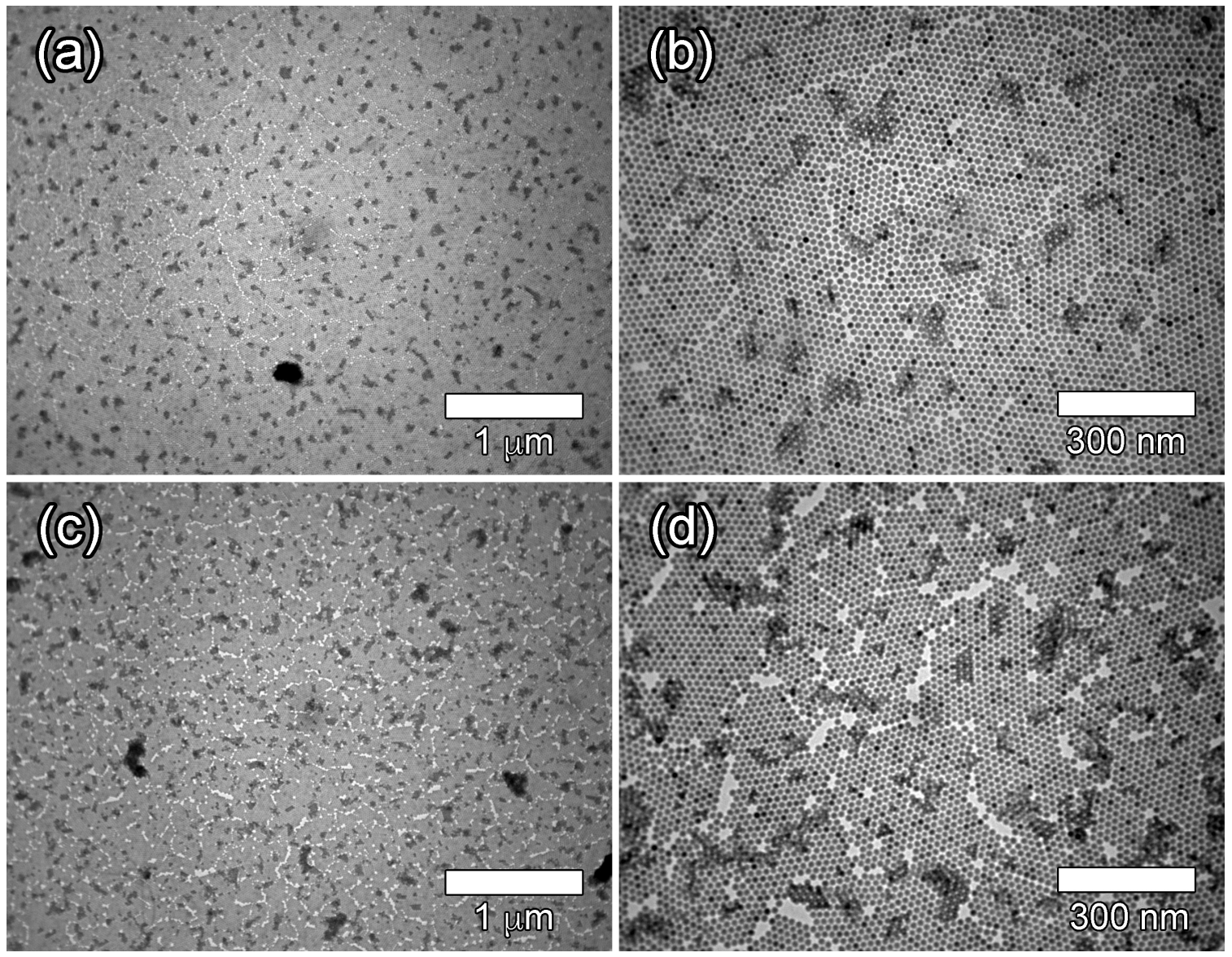

Figure S6. TEM images of $13.2 \mathrm{~nm}$ iron oxide NP monolayer and, in a few places, bilayer formed by using the (a),(b) benzene/DMSO and (c),(d) fluorobenzene/DMSO miscible solvent systems.

The miscible solvent pair method was also tested for other particles, e.g. $4 \mathrm{~nm}$ CdSe quantum dots (QDs) (using the toluene/DMSO miscible solvent pair) and $5 \mathrm{~nm}$ iron oxide NPs (benzene/DMSO miscible solvent pair). NPs still form thin layers on the liquid 
surface, but with a larger double layer percentage ( $>50 \%$ for $4 \mathrm{~nm}$ CdSe QDs and $>30 \%$ for $5 \mathrm{~nm}$ iron oxide NP), than for $13.2 \mathrm{~nm}$ iron oxide NPs (See Fig. S7). The small NP sizes might be one of the reasons for this higher double layer coverage. In these cases, the diameters of the NPs are comparable with the ligand shell thickness, so the first layer is randomly close-packed (as seen for these $4 \mathrm{~nm}$ CdSe QDs and $5 \mathrm{~nm}$ iron oxide NPs) due to the soft ligand shell structure. Therefore, the second layer might be in contact the liquid surface through the vacancies formed in the first layer.
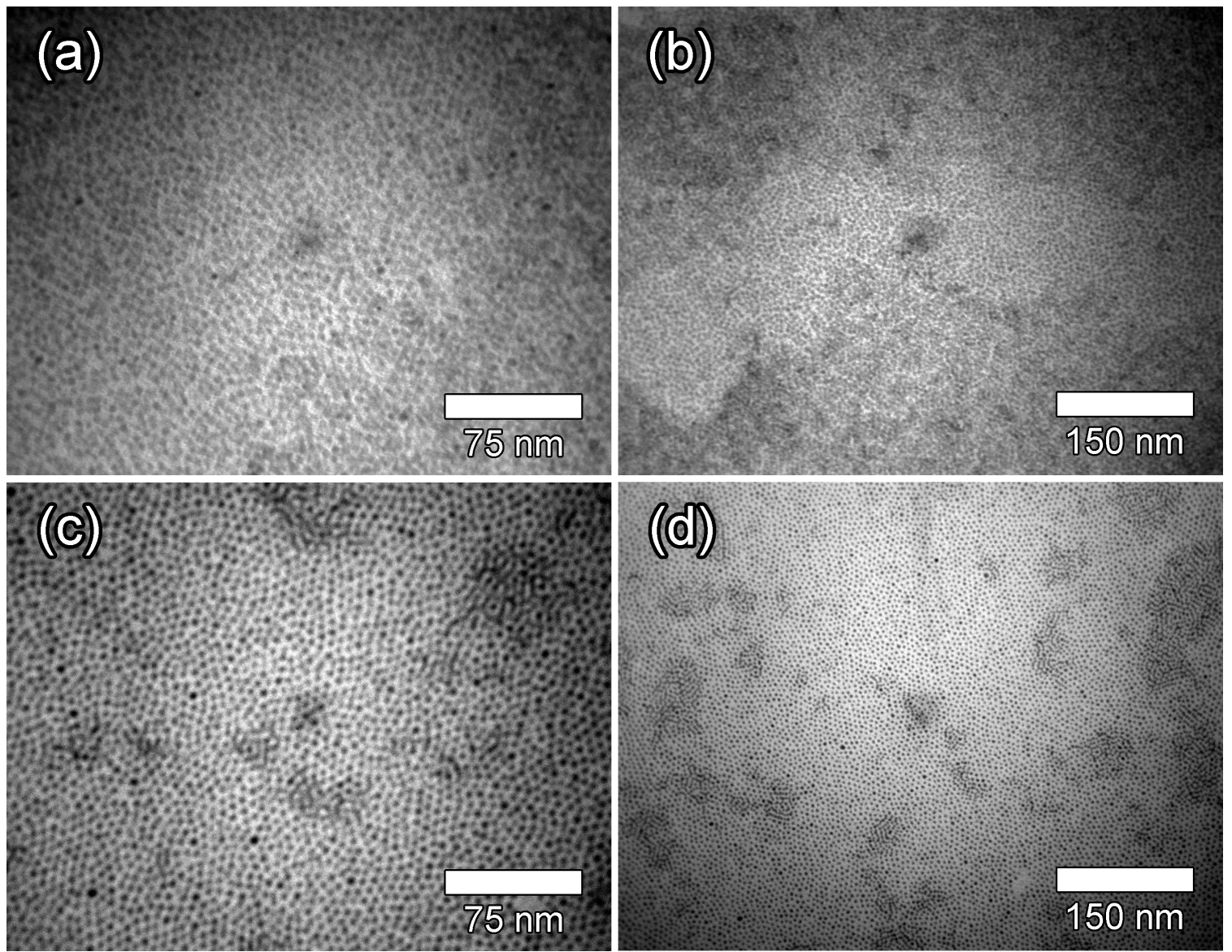

Figure S7. TEM images of (a),(b) $4 \mathrm{~nm}$ CdSe NP monolayer and, in places, bilayer formed using the toluene/DMSO miscible solvent pair system and (c),(d) $5 \mathrm{~nm}$ iron oxide NP monolayer formed using the benzene/DMSO miscible solvent pair system. 


\section{REFERENCES}

(1) Narayanan, S.; Wang, J.; Lin, X. M. Phys. Rev. Lett. 2004, 93, 135503.

(2) Jiang, Z.; Lin, X.; Sprung, M.; Narayanan, S.; Wang, J. Nano Lett. 2010, 10, 799803.

(3) Dutta, P.; Sinha, S. K. Phys. Rev. Lett. 1981, 47, 50-53

(4) Pieranski, P. Phys. Rev. Lett. 1980, 45, 569-572. 